\title{
Estudo Comparativo da Biodisponibilidade Relativa de Duas Formulações de Glimepirida em Voluntários Sadios de Ambos os Sexos Após Administração de Uma Dose Única de Um Comprimido de $4 \mathrm{mg}$
}

\section{artigo original}

\author{
Ney Carter do Carmo Borges \\ YARA DEL ANTONIO TAVEIRA \\ JOSIMARA A. MAZUCHELI \\ ANDRÉ LUIZ HADDAD \\ Rafael E. B. AstigarRaga \\ RONILSON AGNALDO MORENO
}

Synchrophar Assessoria e

Desenvolvimento de Projetos Clínicos S/S Ltda. (NCCB, YDAT,

JAM \& RAM), Departamento de

Clínica Médica (NCCB) e

Departamento de Farmacologia

(RAM), Faculdade de Ciências

Médicas, Universidade Estadual de Campinas (UNICAMP),

Campinas, SP; e MAGABI

Pesquisas Clínicas $e$

Farmacêuticas Ltda. (ALH), São

Paulo, SP.

Recebido em 11/05/06

Revisado em 11/12/06

Aceito em 20/12/06
RESUMO

Objetivo: Comparar a biodisponibilidade de duas formulações de glimepirida em 26 voluntários sadios de ambos os sexos. Material e métodos: $\mathrm{O}$ estudo foi aberto, cruzado e randomizado com dois períodos e wash out de 14 dias. As amostras foram obtidas em um intervalo de 48 horas. As concentrações de glimepirida foram analisadas por HPLC MS/MS. Das curvas de concentração de glimepirida no plasma versus tempo, foram obtidos os seguintes parâmetros farmacocinéticos: $\mathrm{ASC}_{(0-\mathrm{t})}$, $\mathrm{ASC}_{(0-\infty)}, \mathrm{C}_{\max }, \mathrm{K}_{\mathrm{e}}, \mathrm{T}_{\max }$ e $\mathrm{T}_{1 / 2}$. Resultados: $\mathrm{A}$ razão entre as média geométricas de Glimepirida/Amaryl ${ }^{\circledR} 4 \mathrm{mg}$ foi de $102,35 \%$ para $\mathrm{ASC}_{(0-\mathrm{t})}$; $102,35 \%$ para $\mathrm{ASC}_{(0-\infty)}$ e $99,31 \%$ para $\mathrm{C}_{\text {max }}$. Os intervalos de confiança de $90 \%$ (IC 90\%) foram de 92,62-109,55\%; 95,62-109,55\% e 88,60-111,32\%, respectivamente. Conclusão: Como o IC $90 \%$ para $\mathrm{C}_{\max }, \mathrm{ASC}_{(0-\mathrm{t})}$ e $\mathrm{ASC}_{(0-\infty)}$ estava dentro do intervalo de $80-125 \%$, concluiu-se que ambas as formulações foram bioequivalentes de acordo com o grau e a extensão de sua absorção. (Arq Bras Endocrinol Metab 2007;51/6:950-955)

Descritores: Glimepirida; Diabetes; Bioequivalência; Cromatografia líquida

\section{ABSTRACT}

Comparison Study of Two Glimepiride Formulations Bioavailability in Healthy Volunteers of Both Sexes After a Single Dose Administration.

Objective: To compare the bioavailability of two glimepiride 4-mg tablet formulation in 26 healthy volunteers of both sexes. Material and methods: The study was conducted open with randomized two-period crossover design and a 14-day washout period. Samples were obtained over a 48-hour interval. Glimepiride concentrations were analyzed by LC-MS-MS. From the glimepiride plasma concentration versus time curves the following pharmacokinetic parameters were obtained: $\mathrm{AUC}_{(0-\text { last })}, \mathrm{AUC}_{(0-\mathrm{t})}, \mathrm{AUC}_{(0-\infty)}, \mathrm{K}_{\mathrm{e}^{\prime}}, \mathrm{T}_{1 / 2}$, $\mathrm{C}_{\text {max }}$ and $\mathrm{T}_{\text {max }}$. Results: Geometric mean of Glimepirida/Amaryl ${ }^{\circledR} 4 \mathrm{mg}$ was $102.35 \%$ for $A \cup C_{(0-t)}, 102.35 \%$ for $A \mathrm{CC}_{(0-\infty)}$ and $99.31 \%$ for $\mathrm{C}_{\max }$. The 90\% Cl was 92.62-109.55\%; 95.62-109.55\% e 88.60-111.32\%, respectively. Conclusion: Since the $90 \% \mathrm{Cl}$ for both $\mathrm{C}_{\text {max }}, \mathrm{AUC}_{(0-\mathrm{t})}$, and $\mathrm{AUC}_{(0-\infty)}$ were within the interval of $80-125 \%$, it was concluded that both formulations were bioequivalent, according to both the rate and extent of absorption. (Arq Bras Endocrinol Metab 2007;51/6:950-955)

Keywords: Glimepiride; Diabetes; Bioequivalence; Liquid chromatography 
$\mathrm{O}$

DIABETES MELLITUS TIPO 2 (DM2) é, atualmente, uma das principais causas de morte no mundo, sendo considerado uma doença epidêmica. Estima-se que o DM2 seja a causa de 1 em cada 20 mortes e, em adultos com idade entre 35 e 64 anos, 1 em cada 10 mortes (1). Espera-se que até 2025 a incidência de DM2 tenha um crescimento de $35 \%$ em países em desenvolvimento (2).

O DM2 é uma síndrome de etiologia múltipla, decorrente da falta de insulina e/ou da incapacidade da insulina em exercer adequadamente seus efeitos. Caracteriza-se por hiperglicemia crônica, freqüentemente acompanhada de dislipidemia, hipertensão arterial e disfunção endotelial (3).

O tratamento comumente utilizado para o DM2 consiste em dietas e exercícios físicos adequados. Entretanto, nos casos em que mesmo o tratamento usual não resulta em regularidade no metabolismo, faz-se necessário o uso de medicamentos hipoglicemiantes orais (4).

Dentre estes tipos de medicamentos, atualmente dispõem-se de cinco principais classes distintas: sulfoniluréias, biguanidas, inibidores da $\alpha$-glicosidase, tiazolinedionas e meglitinidas (5).

\section{GLIMEPIRIDA}

A glimepirida é um fármaco que faz parte do grupo da sulfoniluréias. Sua fórmula estrutural é (6):



A glimepirida caracteriza-se por estimular a secreção de insulina, ligando-se a um receptor específico na célula $\beta$, que determina o fechamento dos canais de potássio dependentes de ATP, resultando em despolarização da célula. $\mathrm{O}$ influxo de cálcio secundário à despolarização causa liberação de insulina, que potencializa a ação insulínica no tecido muscular, adiposo e no fígado, diminuindo a produção hepática de glicose (7).

\section{OBJETIVO}

O objetivo deste estudo foi comparar a biodisponibilidade relativa de duas formulações do comprimido de $4 \mathrm{mg}$ de glimepirida em 26 voluntários sadios de ambos os sexos, após ingestão de 1 comprimido em jejum.

\section{MÉTODO}

O estudo foi aberto, randomizado, cruzado, com dois tratamentos e dois períodos, nos quais 26 voluntários sadios (13 homens e 13 mulheres) receberam um comprimido referência ou um teste dependendo da aleatorização a que foram submetidos. As administrações foram precedidas de jejum e tiveram um intervalo de 14 dias entre elas. Os confinamentos tiveram duração de aproximadamente 24 horas cada.

Para análise farmacocinética, foram utilizadas amostras de plasma, sendo realizadas 36 coletas de amostras por voluntário, sendo $18 \mathrm{em}$ cada período de internação.

Para efeito de segurança dos voluntários, foram verificados sinais vitais (pressão arterial, freqüência cardíaca e temperatura) e foram realizados questionamentos para certificação de bem estar geral.

\section{FORMULAÇÕES}

As seguintes formulações foram empregadas: comprimido de glimepirida $4 \mathrm{mg}$ (número de lote 001/03), produzido pela Eurofarma Laboratórios Ltda., como formulação teste e comprimido de Amaryl ${ }^{\circledR} 4$ mg (número de lote 203126), produzido pela Aventis Pharma Ltda., como formulação referência.

\section{CONDUÇÃO CLÍNICA}

Os 26 voluntários foram selecionados mediante comprovação de sua higidez por meio de consulta clínica, submissão a eletrocardiograma e exames laboratoriais.

Após comprovação da higidez, foi realizada uma explanação sobre o estudo e as implicações da participação no mesmo. O protocolo foi aprovado pelo Comitê de Ética da Unicamp e todos os voluntários assinaram um Termo de Consentimento Livre e Esclarecido, declarando estarem cientes de seus direitos e deveres em relação à pesquisa.

$\mathrm{Na}$ noite de confinamento de cada um dos períodos, foi oferecido um jantar de cardápio prédefinido aos voluntários. Os mesmos cumpriram um jejum alimentar de pelo menos 10 horas e um jejum para líquidos de pelo menos 6 horas anteriores à administração da medicação.

A administração da medicação teve início às $7 \mathrm{~h}$ da manhã seguinte ao confinamento. Cada voluntário ingeriu a medicação a que foi designado com $200 \mathrm{~mL}$ de água mineral sem gás e na temperatura ambiente. Após a ingestão, foi cumprido um jejum alimentar de 4 horas. Um desjejum, um almoço, um lanche da tarde e um 
jantar padronizados foram consumidos depois de aproximadamente 4, 6, 8 e 11 horas da administração do medicamento, respectivamente. Não foi permitida a ingestão de nenhum outro alimento durante o período de confinamento. A ingestão de líquidos obedeceu ao seguinte horário: $200 \mathrm{~mL}$ de água após 2 horas de ingestão da medicação e, após almoço, ingestão ad libitum. Os voluntários permaneceram no confinamento por um período total de 24 horas em cada internação.

As pressões arteriais sistólica e diastólica (medida de forma não invasiva com um esfigmomanômetro), a freqüência cardíaca e a temperatura foram registradas anteriormente e em intervalos pré-determinados após a administração das formulações.

As coletas de amostras de sangue ocorreram nos seguintes horários após a administração dos fármacos (hh:mm): 00:00, 00:15, 00:30, 00:45, 01:00, 01:30, 02:00, 02:30, 03:00, 04:00, 05:00, 06:00, 08:00h, 10:00, 12:00, 24:00, 48:00.

Todos os procedimentos e alimentos foram iguais para ambos os períodos.

\section{MÉTODO ANALÍTICO}

As amostras de sangue foram centrifugadas a $2.000 \mathrm{~g}$ por 10 min à temperatura ambiente e o plasma separado e armazenado a $-20^{\circ} \mathrm{C}$ até que fosse avaliado com relação ao conteúdo de glimepirida. A glimepirida e a tolazamida, utilizada como padrão interno na quantificação da glimepirida, foram extraídas do plasma dos voluntários por extração líquido-líquido.

A $300 \mu \mathrm{L}$ de cada amostra de plasma foram adicionados $25 \mu \mathrm{L}$ do padrão interno tolazamida $(2 \mu \mathrm{g} / \mathrm{mL})$, $500 \mu \mathrm{L}$ de solução saturada de cloreto de sódio e $1000 \mu \mathrm{L}$ de acetato de etila. Após agitação por $5 \mathrm{~min}$, os tubos foram centrifugados ( 5 min a $14.000 \mathrm{rpm}$ ) e $800 \mu \mathrm{L}$ do sobrenadante foram transferidos para um outro tubo limpo. O solvente restante no sobrenadante transferido foi, então, evaporado usando um fluxo de ar comprimido a $37^{\circ} \mathrm{C}$ e os resíduos dissolvidos em $300 \mu \mathrm{L}$ de acetonitrila. $250 \mu \mathrm{L}$ desta solução foram, então, transferidos para inserts de vidro descartável e alíquotas de $8 \mu \mathrm{L}$ foram injetadas no sistema cromatográfico.

As concentrações plasmáticas de glimepirida foram medidas através de um método sensível de cromatografia líquida de alta eficiência acoplada à espectrometria de massa (HPLC MS/MS). O espectrômetro de massas utilizado foi o Micromass Quattro Micro equipado com um auto injetor Shimadzu SIL 10 ADVP. O software utilizado foi o Micromass Mass Lynx 3.5.

A glimepirida foi extraída do plasma dos voluntários por extração líquido-líquido. Os compostos foram eluídos em um pequeno volume de fase móvel, cuja alíquota foi analisada por ionização positiva e utilizando o monitoramento do íon selecionado (MRM).
O padrão interno utilizado para quantificação da glimepirida foi a tolazamida.

Os cálculos foram baseados em função da curva de calibração construída para o fármaco analisado, utilizando os padrões de calibração pré-estabelecidos. Estas razões foram calculadas através da razão entre as áreas dos picos da glimepirida e do padrão interno tolazamida. Para as quantificações foram obtidas as razões das áreas dos picos glimepirida/tolazamida. As concentrações plasmáticas das amostras foram derivadas a partir da equação linear da reta, obtida com a curva padrão (concentração de glimepirida em função das áreas).

\section{ANÁLISE FARMACOCINÉTICA E ESTATÍSTICA}

A análise farmacocinética foi realizada com apoio dos softwares Microsoft Excel versão 7.0 e SAS ${ }^{\circledR}$ (Statistical Analysis System) versão 8.2. Os parâmetros determinados foram $\mathrm{ASC}_{(0-\mathrm{t})}, \mathrm{ASC}_{(0-\infty)}$ (calculados pelo método trapezoidal), $\mathrm{K}_{\mathrm{e}}, \mathrm{T}_{1 / 2}, \mathrm{C}_{\max } \mathrm{e} \mathrm{T}_{\max }$.

A concentração máxima no plasma observada $\left(\mathrm{C}_{\max }\right)$ e o tempo necessário para atingir esta concentração $\left(\mathrm{T}_{\max }\right)$ foram obtidos diretamente das curvas. A área sob a curva (ASC) de concentração de glimepirida no plasma vs. tempo de 0 a 48 h $\left(\right.$ ASC $\left._{0-\mathrm{t}}\right)$ foi calculada aplicando-se a regra linear trapezoidal. A extrapolação dessa área ao infinito $\left(\mathrm{ASC}_{0-\infty}\right)$ foi feita acrescentando-se o valor $\mathrm{C}_{0-\mathrm{t}} / \mathrm{K}_{\mathrm{e}}$ à $\mathrm{ASC}_{0-\mathrm{t}}$ calculada. As análises estatísticas foram conduzidas após transformação logarítmica baseada em modelo aditivo para todos os valores de ASC e $\mathrm{C}_{\max }$. A constante da taxa de eliminação terminal de primeira ordem $\left(\mathrm{K}_{\mathrm{e}}\right)$ foi estimada pelo coeficiente angular da reta de regressão, calculada pelo método dos mínimos quadrados, do logaritmo natural da concentração vs. tempo. $\mathrm{O} \mathrm{T}_{1 / 2}$ foi obtido empregando-se a equação $\left(\mathrm{T}_{1 / 2}=\mathrm{ls}(2) / \mathrm{K}_{\mathrm{e}}\right)$. Foi empregada análise de variância (ANOVA) para o modelo de dois períodos cruzados. O critério para equivalência baseada em biodisponibilidade relativa foi intervalo de confiança de $90 \%$ da razão entra as médias geométricas das ASCs (no que diz respeito à extensão da absorção) e dos $\mathrm{C}_{\max } \mathrm{s}$ (no que diz respeito à velocidade de absorção), relativos ao fármaco, dentro do intervalo de $80-125 \%$ (11). Para a constatação da bioequivalência, foi utilizada a bioequivalência média.

\section{RESULTADOS}

A tabela 1 resume os dados demográficos da população que participou do estudo.

Cada formulação de glimepirida foi bem tolerada nas doses administradas. O estudo transcorreu sem nenhum efeito adverso sério. 
Tabela 1. Análise estatística descritiva dos dados demográficos (altura, peso, índice de massa corpórea e idade) dos 26 voluntários que participaram do estudo.

\begin{tabular}{lcccccc}
\hline & & \multicolumn{4}{c}{ Dados demográficos } \\
Variáveis & $\mathbf{N}$ & Média & Desvio-Padrão & Mínimo & Máximo & Mediana \\
Altura & 26 & 1,67 & 0,07 & 1,57 & 1,82 & 1,66 \\
Peso & 26 & 67,6 & 9,7 & 51,2 & 90,7 & 67,8 \\
IMC & 26 & 24 & 2,61 & 19,16 & 27,8 & 24 \\
Idade & 26 & 29 & 6,23 & 20 & 43 & 27,5 \\
\hline
\end{tabular}

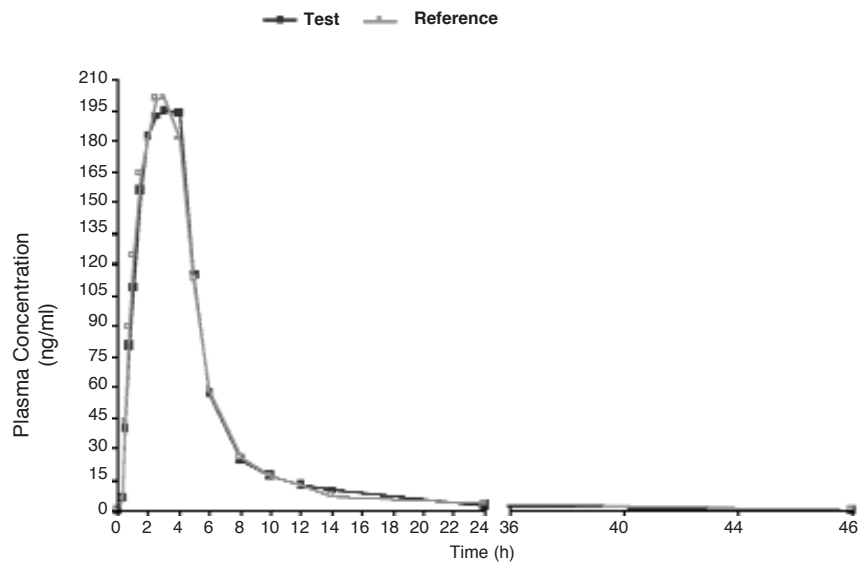

Figura 1. Perfil das concentrações médias de glimepirida no plasma versus tempo, obtido após uma única administração de cada uma das formulações do comprimido de $4 \mathrm{mg}$ de glimepirida.
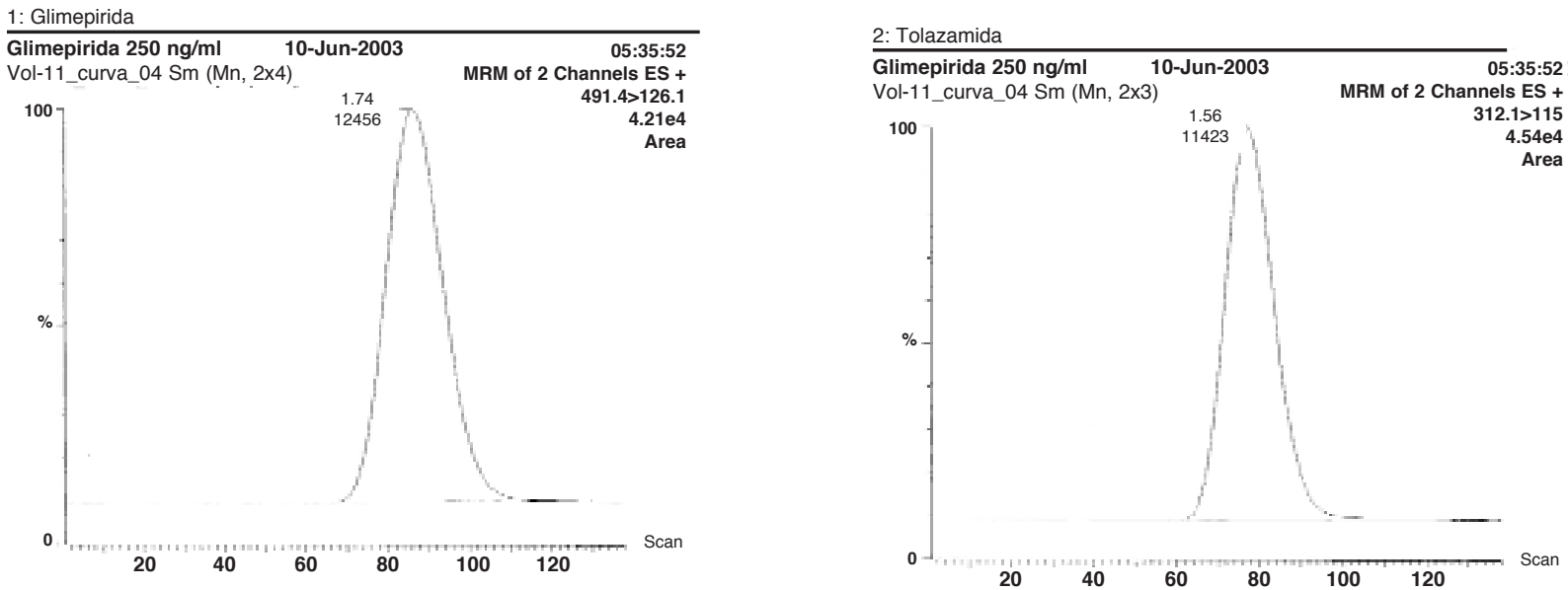

Figura 2. Cromatogramas dos íons selecionados (MRM). A: MRM de 2 canais ES+: 491,4 > 126,1 para a glimepirida. B: MRM de 2 canais ES+: $312,1>115,0$ para a tolazamida. 
Tabela 2. Médias geométricas das razões individuais da $A S C_{0-t}, A S C_{0-u l t}, A S C_{0-\infty}$ e $C_{\text {max }}$ (formulação teste/ referência), os respectivos intervalos de confiança 90\% (IC 90\%) e poder, para a população total de voluntários, população masculina e feminina.

\begin{tabular}{|c|c|c|c|c|}
\hline \multicolumn{5}{|c|}{ Médias geométricas das razões individuais da $A S_{0-t}, A C_{0-u l t}, A C_{0-\infty}$ e $C_{\max }$} \\
\hline Teste/Referência & \multicolumn{4}{|c|}{ Paramétrico $(n=26)$} \\
\hline \multicolumn{5}{|l|}{$\mathrm{n}=26$} \\
\hline $\operatorname{ASC}_{(0-t)} \%$ & 102.47 & $95.74-109.68$ & 0.9997 & 14.39 \\
\hline $\mathrm{ASC}_{(0-\mathrm{ult})} \%$ & 102.97 & $95.36-111.19$ & 0.9983 & 16.29 \\
\hline $\mathrm{ASC}_{(0-\infty)} \%$ & 104.48 & $96.41-113.23$ & 0.9972 & 17.06 \\
\hline $\mathrm{C}_{\max } \%$ & 99.31 & $88.55-111.32$ & 0.9423 & 24.40 \\
\hline & \multicolumn{4}{|c|}{ Paramétrico masculino $(n=13)$} \\
\hline leste/Reterencia & Média Geométrica & IC $90 \%$ & Poder & CV Intra-sujeito (\%) \\
\hline $\mathrm{n}=13$ & 108.36 & $99.80-117.65$ & 0.9947 & 11.68 \\
\hline $\operatorname{ASC}_{(0-t)} \%$ & 110.97 & 101.17-121.72 & 0.9863 & 13.14 \\
\hline $\operatorname{ASC}_{(0-u l t)} \%$ & 108.26 & $99.04-118.34$ & 0.9898 & 12.64 \\
\hline $\mathrm{ASC}_{(0-\infty)} \%$ & 110.06 & $95.32-127.08$ & 0.8289 & 20.56 \\
\hline \multirow[t]{2}{*}{ Teste/Referência } & \multicolumn{4}{|c|}{$\begin{array}{l}\text { Paramétrico feminino }(n=13) \\
\text { Women - Parametric }\end{array}$} \\
\hline & Média Geométrica & IC $90 \%$ & Poder & CV Intra-sujeito (\%) \\
\hline $\mathrm{n}=13$ & 97.95 & $88.11-108.89$ & 0.9641 & 15.06 \\
\hline $\operatorname{ASC}_{(0-t)} \%$ & 96.70 & $86.04-108.69$ & 0.9350 & 16.64 \\
\hline $\operatorname{ASC}_{(0-u l t)} \%$ & 101.62 & 87.82-117.58 & 0.8193 & 20.87 \\
\hline $\mathrm{ASC}_{(0-\infty)} \%$ & 90.95 & $76.15-108.95$ & 0.6738 & 25.54 \\
\hline
\end{tabular}

Tabela 3. Análise estatística dos parâmetros farmacocinéticos médios obtidos dos 26 voluntários após a administração oral de cada formulação de glimepirida $4 \mathrm{mg}$.

\begin{tabular}{lcccc}
\hline \multicolumn{4}{c}{ Análise estatística dos parâmetros farmacocinéticos } \\
& \multicolumn{3}{c}{${\text { Amaryl }{ }^{\circledR}}$} & \multicolumn{2}{c}{ Glimepirida } & \\
& Média & $\mathrm{DP}$ & Média & $\mathrm{DP}$ \\
$\mathrm{ASC}_{(0-\mathrm{t})}([\mathrm{ngh}] / \mathrm{ml})$ & 1083 & 410,9 & 1080 & 331,4 \\
$\mathrm{ASC}_{(0-\infty)}([\mathrm{ngh}] / \mathrm{ml})$ & 1083 & 410,9 & 1080 & 331,4 \\
$\mathrm{C}_{\max }([\mathrm{ngh}] / \mathrm{ml})$ & 241,9 & 81,68 & 238,9 & 71,56 \\
$\mathrm{~K}_{\mathrm{e}}$ & 0,26 & 0,08 & 0,29 & 0,13 \\
$\mathrm{~T}_{1 / 2}(\mathrm{~h})$ & Mediana & $($ Amplitude) & Mediana & (Amplitude) \\
$\mathrm{T}_{\max }(\mathrm{h})$ & 2,63 & 3,35 & 2,34 & 5,75 \\
\hline & 2,50 & 3,00 & 3,00 & 4,00 \\
\hline
\end{tabular}

Treze voluntários apresentaram eventos adversos, sendo 11 queixas clínicas não relacionadas à droga e 2 queixas de cefaléia, que foram classificadas como possivelmente relacionadas à droga, em decorrência do horário de administração da mesma.

Todos os parâmetros clínicos e bioquímicos pós-estudo apresentaram-se sem quaisquer alterações relevantes.

O limite de quantificação (LQ) encontrado foi $5 \mathrm{ng} / \mathrm{ml}$. Os tempos de retenção da glimepirida e da tolazamida foram de 1,74 min e 1,56 min, respectivamente.
A exatidão intracorrida foi de $96,32 \%, 94,23 \%$ e $98,76 \%$, respectivamente. A exatidão intercorridas foi de $98,12 \%, 96,75 \%$ e $98,40 \%$, respectivamente. A exatidão foi obtida pelo valor médio de cada amostra ou lote, em cada nível de concentração dentro de $15 \%$ do valor real (tolerância até $20 \%$ em valores de LQ). O perfil das concentrações médias da glimepirida no plasma versus tempo obtido após uma única administração via oral da formulação teste e da formulação referência do comprimido de $4 \mathrm{mg}$ encontra-se na figura 1 . Os cromatogramas dos íons selecionados para glimepirida e tolazamida são apresentados na figura 2. A tabela 2 mostra os 
parâmetros farmacocinéticos médios obtidos dos 26 voluntários após a administração oral de cada formulação de glimepirida $4 \mathrm{mg}$. A tabela 3 apresenta as médias geométricas das razões individuais da $\mathrm{ASC}_{0-\mathrm{t}}, \mathrm{ASC}_{0 \text {-ult }}$, $\mathrm{ASC}_{0-\infty}$ e $\mathrm{C}_{\text {max }}$, os respectivos intervalos de confiança de $90 \%$ e poder para a análise de bioequivalência das duas formulações (teste versus referência).

\section{DISCUSSÃo}

A glimepirida faz parte de uma classe de fármacos considerada segura, tendo a hipoglicemia como efeito adverso mais comum (4). Neste estudo, a segurança demonstrada pela literatura no uso do fármaco foi comprovada pela ausência de eventos adversos relacionados à administração da glimepirida.

Em um estudo de bioequivalência de duas formulações de glimepirida realizado por Song e cols. (4), em 2005, o resultado encontrado para o LQ foi de 10 $\mathrm{ng} / \mathrm{mL}$, um valor mais alto do que o encontrado na presente pesquisa. Salem e cols. (10) realizaram, em 2003, um estudo de bioequivalência utilizando como metodologia analítica o HPLC MS/MS encontrando um LQ também mais alto que o encontrado neste estudo: $5.0 \mathrm{ng} / \mathrm{ml}$.

Pérez e cols. (12) realizaram, em 2005, um estudo de validação de método analítico para determinação de glimepirida em plasma humano por meio de HPLC/UV, no qual foi encontrado um tempo de retenção de 4,5 min, ou seja, um tempo muito superior ao 1,60 min encontrado nesta pesquisa.

Pode-se inferir, desta maneira, que o método HPLC MS/MS, descrito aqui para a quantificação da droga, está de acordo com os conceitos de alta sensibilidade e especificidade, como demonstram os estudos supra citados.

O pico das concentrações de glimepirida no plasma de ambas as formulações orais e o $\mathrm{T}_{\max }$ foram semelhantes àqueles relatados na literatura (10-13).

Existe uma literatura escassa de estudos de bioequivalência de glimepirida realizados com homens e mulheres, pois os relatos apontam a participação de voluntários do sexo masculino (9). Entretanto, a presente pesquisa demonstra que não há diferenças significativas entre os valores estatísticos encontrados na análise paramétrica de homens e mulheres (tabela 2).

Os resultados estatísticos encontrados (tabela 3 ) indicam que nenhum dos parâmetros usados para determinar a bioequivalência $\left(\mathrm{ASC}_{(0-48 \mathrm{~h})}, \mathrm{ASC}_{(0-\propto)} \mathrm{e}\right.$ $\mathrm{C}_{\max }$ e $\mathrm{T}_{\max }$ ) foi significativamente diferente para os dois fármacos (teste e referência).
Como os IC 90\% para as razões médias de

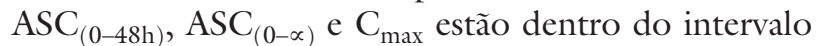
de $80-125 \%$ proposto pelo FDA e pela Anvisa, conclui-se que as duas formulações de glimepirida (comprimido de $4 \mathrm{mg}$ ) são bioequivalentes, tanto pela extensão quanto pela taxa de sua absorção.

\section{REFERÊNCIAS}

1. The prevention of diabetes and its complications. Announcement of a new WHO technical report on the prevention of diabetes and its complications. Available at: <http://www.who.int/diabetes/preventionflyer/en/>. Accessed in October 2006.

2. Passos VMA, Barreto SM, Diniz LM, Lins-Costa M. Type 2 diabetes: prevalence and associated factors in a Brazilian community - the Bambuí health and aging study. São Paulo Med J 2005;123(2).

3. Sociedade Brasileira de Diabetes. Consenso Brasileiro Sobre Diabetes 2002. Diagnóstico e classificação do diabetes melito e tratamento do diabetes melito do tipo 2. Rio de Janeiro: Diagraphic Editora, 2003.

4. Song YK, Maeng JE, Hwang HR, Park JS, Kim BC, Kim JK, et al. Determination of glimepiride in human plasma using semi-microbore high performance liquid chromatography with column-switching. J Chromatogr B Analyt Technol Biomed Life Sci 2004;810(1):143-9.

5. World Health Organization - WHO. Diabetes Programme Country and regional data. Available at: <http:// www.who.int/diabetes/facts/world_figures/en/index.ht $\mathbf{m l}>$. Accessed in October 2006

6. PDR Physicians Desk Reference ${ }^{\circledR}, 2006$.

7. Young EC, Pires MLE, Lima MBC. Cad Bras Med 2001; XIV(1-4).

8. UpToDate. Putting clinical information into practice. Available at: <http://www. uptodate.com>. Accessed in October 2006.

9. Lee $\mathrm{H}-\mathrm{W}$, et al. Bioequivalence of Mepiril tablet to Amaryl tablet (Glimepiride $2 \mathrm{mg}$ ) by liquid chromatography/electrospray tandem mass spctrometry. J Korean Pharm Sci 2005;35(4):287-93.

10. Salem II, Idrees J, AI Tamimi JI. Determination of glimepiride in human plasma by liquid chromatography-electrospray ionization tandem mass spectrometry. J Chromatogr B Technol Biomed Life Sci 2004;799(1):103-9.

11. Pistos C, Astraka C, Kalovidouris M, Vassilopoulos E, Koutsopoulou M. Bioequivalence evaluation of two brands of glimepiride $4 \mathrm{mg}$ tablets in healthy subjects. Int J Clin Pharmacol Ther 2005;43(4):203-8.

12. Pérez $M$, et al. Validación del método analítico para la determinación de glimepirida em plasma humano por HPLC/UV com adición de estándar empleando glibenclamida como estándar interno. Ars Pharm 2005;46(4):411-27.

13. Dotsikas Y, Kousoulos C, Tsatsou G, Loukas YL. Development of a rapid method for the determination of glimepiride in human plasma using liquid-liquid extraction based on 96well format micro-tubes and liquid chromatography/tandem mass spectrometry. Rapid Commun Mass Spectrom 2005;19(14):2055-61.

Endereço para correspondência:

Ronilson A. Moreno

Rua Dr. Cândido Gomide 38

13070-200 Campinas, SP

Fax: (19) 3236-8014

E-mail: moreno@synchrophar.com 